\title{
Systemic inflammatory response syndrome in adult patients with nosocomial bloodstream infections due to enterococci Katharine Bar ${ }^{1}$, Hilmar Wisplinghoff ${ }^{2}$, Richard P Wenzel ${ }^{1}$, Gonzalo ML Bearman ${ }^{1}$ and Michael B Edmond*1
}

\author{
Address: ${ }^{1}$ Department of Internal Medicine, Virginia Commonwealth University, Richmond, VA, USA and ${ }^{2}$ Institute for Medical Microbiology, \\ Immunology and Hygiene, University of Cologne, Cologne, Germany \\ Email: Katharine Bar - katharinebar@gmail.com; Hilmar Wisplinghoff - hwisplinghoff@hotmail.com; Richard P Wenzel - rwenzel@vcu.edu; \\ Gonzalo ML Bearman - gbearman@vcu.edu; Michael B Edmond*-medmond@vcu.edu \\ * Corresponding author
}

Published: 26 September 2006

BMC Infectious Diseases 2006, 6:145 doi:10.1186/147/-2334-6-145
Received: 3I May 2006

Accepted: 26 September 2006

This article is available from: http://www.biomedcentral.com/I47I-2334/6//45

(C) 2006 Bar et al; licensee BioMed Central Ltd.

This is an Open Access article distributed under the terms of the Creative Commons Attribution License (http://creativecommons.org/licenses/by/2.0), which permits unrestricted use, distribution, and reproduction in any medium, provided the original work is properly cited.

\begin{abstract}
Background: Enterococci are the third leading cause of nosocomial bloodstream infection (BSI). Vancomycin resistant enterococci are common and provide treatment challenges; however questions remain about VRE's pathogenicity and its direct clinical impact. This study analyzed the inflammatory response of Enterococcal BSI, contrasting infections from vancomycin-resistant and vancomycin-susceptible isolates.
\end{abstract}

Methods: We performed a historical cohort study on 50 adults with enterococcal BSI to evaluate the associated systemic inflammatory response syndrome (SIRS) and mortality. We examined SIRS scores 2 days prior through 14 days after the first positive blood culture. Vancomycin resistant ( $\mathrm{n}$ $=17)$ and susceptible infections $(n=33)$ were compared. Variables significant in univariate analysis were entered into a logistic regression model to determine the affect on mortality.

Results: $60 \%$ of BSI were caused by E. faecalis and $34 \%$ by E. faecium. $34 \%$ of the isolates were vancomycin resistant. Mean APACHE II (A2) score on the day of BSI was 16. Appropriate antimicrobials were begun within 24 hours in $52 \%$. Septic shock occurred in $62 \%$ and severe sepsis in an additional $18 \%$. Incidence of organ failure was as follows: respiratory $42 \%$, renal $48 \%$, hematologic $44 \%$, hepatic $26 \%$. Crude mortality was $48 \%$. Progression to septic shock was associated with death $(O R 14.9, \mathrm{p}<.00 \mathrm{I})$. There was no difference in $\mathrm{A} 2$ scores on days $-2,-\mathrm{I}$ and 0 between the VRE and VSE groups. Maximal SIR (severe sepsis, septic shock or death) was seen on day 2 for VSE BSI vs. day 8 for VRE. No significant difference was noted in the incidence of organ failure, 7-day or overall mortality between the two groups. Univariate analysis revealed that AP2> 8 at BSI onset, and respiratory, cardiovascular, renal, hematologic and hepatic failure were associated with death, but time to appropriate therapy $>24$ hours, age, and infection due to VRE were not. Multivariate analysis revealed that hematologic $(O R 8.4, p=.025)$ and cardiovascular failure (OR 7.5, $\mathrm{p}=032)$ independently predicted death.

Conclusion: In patients with enterococcal BSI, (I) the incidence of septic shock and organ failure is high, (2) patients with VRE BSI are not more acutely ill prior to infection than those with VSE BSI, and (3) the development of hematologic or cardiovascular failure independently predicts death. 


\section{Background}

Over the past two decades, enterococcal bloodstream infections (BSI) have become increasingly pervasive. They account for a significant proportion of nosocomial BSI, and are now the third most common cause of nosocomial BSI [1]. Vancomycin resistance is common, found in approximately $2 \%$ of E. faecalis and $60 \%$ of E. faecium strains isolated in the United States [1]. Despite the increasing frequency of vancomycin-resistant enterococcal (VRE) infections, questions remain about VRE's pathogenicity and its direct clinical impact.

Patients with VRE BSI are often critically ill; it is difficult to determine whether the associated high mortality is directly attributable to the infecting organism or a marker of the patients' severe illness. Many studies have attempted to address this question, with conflicting results. Several have attributed high levels of morbidity and mortality to VRE [2-7]. Others have been unable to link increased mortality to vancomycin-resistance, demonstrating that vancomycin resistance is more of a marker of severe disease than a direct cause of poor outcome [811]. Most of the studies investigating enterococcal bloodstream infections (BSI), analyze predisposing factors and outcome measures, without closely examining the clinical course of patients through the infection. This study was conducted to evaluate the inflammatory response, clinical course and outcome of nosocomial bloodstream infections due to enterococci, as well as the effects of vancomycin resistance.

\section{Methods \\ Setting}

The Virginia Commonwealth University Medical Center (VCUMC) is an 820-bed, tertiary care facility in Richmond, Virginia. The hospital has nine intensive care units (ICUs), including pediatric ICUs and a burn unit. Approximately 30,000 patients are admitted annually. The study was approved by our Institutional Review Board.

\section{Study design}

Using the Surveillance and Control of Pathogens of Epidemiological Importance (SCOPE) database [1], we identified all patients diagnosed with enterococcal BSI at VCUMC from November 2000 through December 2002. Patients were considered to have had an enterococcal BSI if they had at least one blood culture positive for this organism. Only monomicrobial BSI were included. Clinical data were retrospectively collected, including age, gender, location of the patient, clinical service, duration of hospitalization prior to onset of BSI, predisposing clinical conditions, the bloodstream pathogen and its antimicrobial susceptibilities. Predisposing clinical conditions included neutropenia (defined as an absolute neutrophil count of less than $500 / \mu \mathrm{l}$ ), peritoneal or hemodialysis, mechanical ventilation, total parenteral nutrition, transfusion, antibiotic use, ICU stay, and intravascular catheters (i.e., central or peripheral intravenous catheters). Adverse outcomes that occurred during the hospital stay were recorded, including organ system failure and death. Accordingly, we evaluated only in-hospital mortality. Patients discharged from the hospital in less than seven days from the onset of BSI were assumed to be alive at seven days. The clinical condition of the patient was classified daily according to the systemic inflammatory response syndrome (SIRS) criteria (SIRS, sepsis, severe sepsis or septic shock) and APACHE II scores from two days prior through 14 days after onset of BSI. Patients who had nosocomial BSI due to vancomycin-resistant enterococci were compared with patients who had nosocomial BSI due to vancomycin-susceptible enterococci.

\section{Definitions}

The date the positive blood culture was drawn was deemed Day 0 in the tracking of BSI. The patients' physiologic condition prior to the BSI and on the day of BSI were assessed using the APACHE II score. A cut-point of greater than 18 was used to stratify the severity of clinical condition. This methodology was used to be consistent with our previous studies in this area. The clinical condition of each patient during the bloodstream infection was classified daily as SIRS, sepsis, severe sepsis or septic shock using criteria previously published by the American College of Chest Physicians/Society of Critical Care Medicine (ACCP/SCCM). [12] Systemic inflammatory response syndrome (SIRS) was defined as two or more of the following: (1) temperature $>38^{\circ} \mathrm{C}$ or $<36^{\circ} \mathrm{C}$, (2)heart rate $>90$ beats per minute, (3) respiratory rate $>20$ breaths per minute or a $\mathrm{PaCO}_{2}<32 \mathrm{mmHg}$, or (4) white blood cell count $>12 \times 10^{9} / \mathrm{L}$ or $<4 \times 10^{9} / \mathrm{L}$ or the presence of more than $10 \%$ immature neutrophils. Sepsis was defined as SIRS associated with Enterococcus isolated from at least one blood culture. Sepsis associated with organ dysfunction, hypotension or systemic manifestations of hypoperfusion constituted severe sepsis. Septic shock was defined as sepsis associated with hypotension unresponsive to intravenous fluid challenge or the requirement of a vasopressor agent. SIRS 0-4, severe sepsis, septic shock and death were used as mutually exclusive categories, with SIRS $0,1,2,3$, and 4 representing the sum of the clinical criteria used to score SIRS. The presence of organ system failure was assessed using the criteria described by Fagon [14]. Nosocomial infection and sources of infection were defined according to Centers for Disease Control and Prevention (CDC) criteria [14]. Adequate empiric antimicrobial therapy was defined as treatment administered within 24 hours of the positive blood culture with an agent to which the enterococcal isolate was susceptible. Per CDC definitions, if the patient had a central venous catheter present at the time of BSI and did not have an infection 
with the same organism at a different site, the infection was determined to be central venous catheter-related.

\section{Microbiological methods}

Blood cultures were processed at the VCUMC clinical laboratory. After identifying to Enterococcus genus, a Strep API kit was used to determine species. Minimum inhibitory concentrations were determined using the E-test. Results were confirmed using a microbroth dilution method according to the Clinical and Laboratory Standards Institute. Vancomycin resistance was defined as an MIC $\geq 32$ $\mu \mathrm{g} / \mathrm{mL}$.

\section{Statistical analysis}

Results were expressed as a mean $\pm \mathrm{SD}$, or as a proportion of the total number of patients or isolates. For continuous variables, mean values were compared using two sample t-tests for independent samples. Differences in proportions were compared using a Chi-square test or Fisher's Exact Test, as appropriate. Mean values are reported \pm SD. All tests of significance were two-tailed, with $\alpha$ set at 0.05 . Independent predictors of the outcome of BSI were identified by means of stepwise logistic regression analysis, with a limit for entering and removing variables at 0.05 . Our dependent variable was mortality, either 7-day or total in-hospital. All statistical analyses were done using SPSS software (SPSS Inc., Chicago, IL, USA).

\section{Results}

\section{Study populations and patient characteristics}

During the study period, a total of 330 nosocomial enterococcal BSIs were identified. Twenty-two were in pediatric patients (age $<18$ years), 245 were polymicrobial, and 13 had incomplete records. The remaining 50 monomi- crobial BSIs caused by Enterococcus spp were analyzed (Table 1). Patients included in the study had a mean age of 52 years, and $50 \%$ of patients were female.

Forty-three (86\%) patients had primary, catheter-related infections. Seven patients had secondary BSI, with four stemming from urinary tract infections and one each from an infected wound, an infected peritoneal fluid and an abscess. Thirty-seven (74\%) of the nosocomial BSIs occurred in the ICU setting. The patients were nearly evenly divided among internal medicine and surgery services $(48 \%$ were on the internal medicine service, $44 \%$ were on the surgery service, and $8 \%$ were on the hematology-oncology service). The admitting diagnoses were classified as hepatic $(22 \%)$, cardiac $(18 \%)$, cancer $(12 \%)$, pulmonary $(12 \%)$, GI $(10 \%)$, trauma $(10 \%)$, neurologic $(8 \%)$ and transplantation (6\%).

Among potential factors predisposing to BSI, intravascular devices were the most common, with 44 patients (88\%) having central venous catheters. Prior to BSI, thirty patients $(60 \%)$ required mechanical ventilation, while 26 patients (52\%) received blood products, 9 patients (18\%) underwent hemodialysis, 6 patients $(12 \%)$ received total parenteral nutrition and 3 patients $(6 \%)$ were neutropenic. The mean length of hospital stay prior to BSI was 21 days. Five patients $(10 \%)$ were discharged within seven days of onset of BSI. Mean APACHE II score on day of BSI was 16.

\section{Microbiological features}

Of the 50 enterococcal isolates, thirty $(60 \%)$ were $E$. faecalis and seventeen (34\%) were E. faecium. The remaining three isolates were E. durans, E. gallinarum and E. casseli-

Table I: Patient characteristics stratified by resistance pattern of infecting organism (VRE vs. VSE) and underlying severity of illness before infection.

\begin{tabular}{|c|c|c|c|c|c|}
\hline & Total $(n=50)$ & VSE $(n=33)$ & $\operatorname{VSE}(n=17)$ & $A P \leq 18(n=38)$ & $A P 2>18(n=12)$ \\
\hline Mean age (years) & 52 & 53 & 49 & $48 *$ & $63 *$ \\
\hline Women & $50 \%$ & $48 \%$ & $53 \%$ & $55 \%$ & $33 \%$ \\
\hline Mean LOS prior to BSI (days) & 21 & $16^{*}$ & $30 *$ & 22 & 18 \\
\hline Mechanical ventilation & $60 \%$ & $54 \%$ & $71 \%$ & $55 \%$ & $75 \%$ \\
\hline Hemodialysis & $18 \%$ & $12 \%$ & $29 \%$ & $13 \%$ & $33 \%$ \\
\hline TPN & $12 \%$ & $6 \%$ & $23 \%$ & $16 \%$ & $0 \%$ \\
\hline Transfusion & $52 \%$ & $48 \%$ & $59 \%$ & $50 \%$ & $58 \%$ \\
\hline Prior antibiotics & $84 \%$ & $78 \%$ & $94 \%$ & $86 \%$ & $75 \%$ \\
\hline ICU care & $74 \%$ & $73 \%$ & $76 \%$ & $76 \%$ & $67 \%$ \\
\hline Central venous catheter & $88 \%$ & $88 \%$ & $88 \%$ & $87 \%$ & $92 \%$ \\
\hline Neutropenia & $6 \%$ & $3 \%$ & $12 \%$ & $8 \%$ & $0 \%$ \\
\hline Vancomycin resistance & $34 \%$ & & & $34 \%$ & $23 \%$ \\
\hline $\mathrm{AP} 2>18$ at day 0 & $24 \%$ & $24 \%$ & $23 \%$ & & \\
\hline Mean time to appropriate antimicrobial therapy (days) & 1.4 & 1.3 & 1.7 & $1.6^{*}$ & $0.8^{*}$ \\
\hline
\end{tabular}

(APACHE II scores on day of onset of illness $>18$ or $\leq 18$ )

$* \mathrm{P}<0.05$ 
flavus. Seventeen (34\%) (all E. faecium) isolates were vancomycin resistant. Three of the vancomycin-susceptible isolates and all of the vancomycin resistant isolates were resistant to ampicillin. Length of hospitalization (mean) prior to BSI was longer for those patients with vancomycin-resistant than those with vancomycin-susceptible enterococcal BSIs ( 30 vs. 16 days, $\mathrm{p}<0.05$ ). The mean time to appropriate therapy was 1.4 days, without a statistically significant difference between the VRE and VSE groups.

\section{Clinical course}

Septic shock occurred in $62 \%$ of patients, and severe sepsis in an additional 18\%, (figure 1). The incidence of organ failure was as follows: respiratory $42 \%$, renal $48 \%$, hematologic 44\%, and hepatic 26\%. Seven-day mortality was $18 \%$ and overall crude mortality was $48 \%$, (table 2 ). Progression to septic shock was significantly associated with 7-day mortality (OR 8.5, p = 0.004) and overall mortality (OR 14.9, $\mathrm{p}<0.001$ ). The mean APACHE II score on the day of BSI was 16. The APACHE II score on the day 0 of the BSI was significantly associated with mortality (OR 8.0 for those with APACHE II $>18, \mathrm{p}=0.005$ ) (Table 2).
There was no difference in APACHE II scores on days -2, 1 , and 0 between the VRE and VSE groups. Maximal inflammatory response (MIR; proportion of patients with severe sepsis, septic shock or death) was seen on day 2 for VSE BSI vs. day 8 for VRE BSI and was significantly greater in those with VRE (Figure 3). No significant difference was noted in the incidence of organ failure, 7-day mortality or overall mortality between the two groups. Univariate analysis revealed that respiratory, cardiovascular, renal, hematologic and hepatic failure, as well as APACHE II score $>18$ two days prior to BSI were associated with death, but time to appropriate therapy $>24$ hours, age and infection due to VRE were not.

Variables that were statistically significant in univariate analyses were selected for multiple logistic regression. Multivariate analysis revealed that hematologic failure (OR 8.6, $\mathrm{p}=0.04$ ) and cardiovascular failure (OR 7.6, $\mathrm{p}=$ 0.06 ) independently predicted death.

\section{Discussion}

Our study investigated monomicrobial enterococcal BSI. Of the 308 infections in adult patients analyzed, only

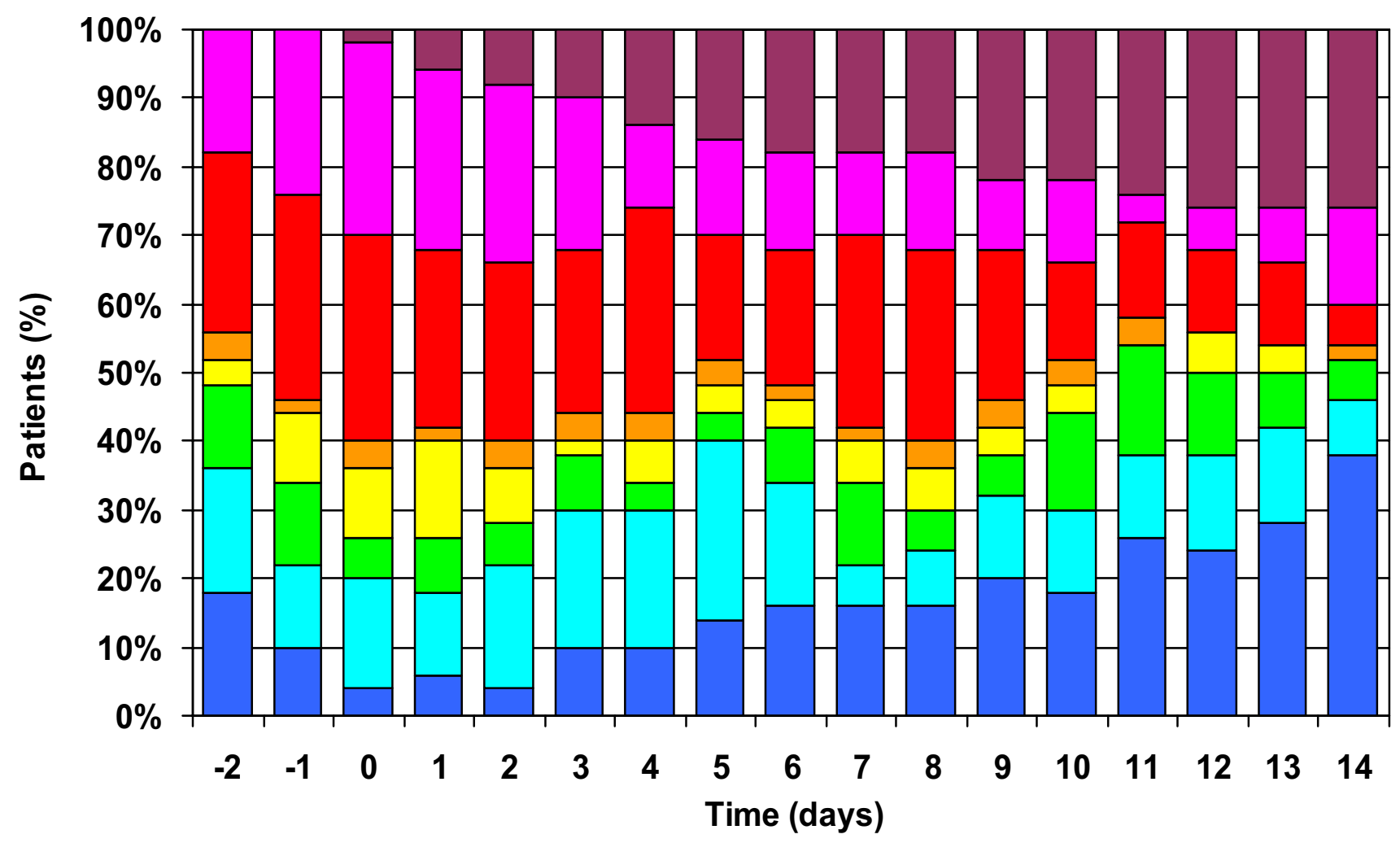

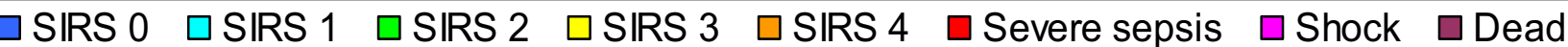

Figure I

Systemic Inflammatory response (SIRS) over time in patients with enterococcal BSI. 
Table 2: Patient outcomes stratified by resistance pattern of infecting organism (VRE vs. VSE) and underlying severity of illness before infection.

\begin{tabular}{|c|c|c|c|c|c|}
\hline & Total $(n=50)$ & VSE $(n=33)$ & $\operatorname{VRE}(n=17)$ & $\mathrm{AP} 2 \leq 18$ & $A P>18$ \\
\hline Respiratory failure & $42 \%$ & $42 \%$ & $41 \%$ & $32 \% *$ & $75 \% *$ \\
\hline Cardiovascular failure & $40 \%$ & $42 \%$ & $35 \%$ & $34 \%$ & $58 \%$ \\
\hline Renal failure & $48 \%$ & $42 \%$ & $59 \%$ & $39 \% *$ & $75 \% *$ \\
\hline Hematologic failure & $44 \%$ & $33 \% *$ & $65 \% *$ & $42 \%$ & $50 \%$ \\
\hline Liver failure & $26 \%$ & $24 \%$ & $29 \%$ & $24 \%$ & $33 \%$ \\
\hline 7-day mortality & $18 \%$ & $15 \%$ & $23 \%$ & $13 \%$ & $33 \%$ \\
\hline Overall mortality & $48 \%$ & $42 \%$ & $59 \%$ & $39 \% *$ & $75 \% *$ \\
\hline
\end{tabular}

(APACHE II scores on day of onset of illness $>18$ or $\leq 18$ )

$* P<0.05$

$16 \%$ (50) of these were monomicrobial, which is lower than in other reports $[5,6]$. The patients in our study were severely ill by several markers. The mean length of hospitalization prior to BSI was long (21 days), which is consistent with nationwide trends [1]. The majority (74\%) were cared for in intensive care settings at the onset of their BSI. They also had significant mortality, with 18\% 7day mortality and $48 \%$ overall mortality. Our patients also had a high level of resistant infecting organisms; 34\% of the enterococcal isolates were resistant to vancomycin. Many studies in the past 15 years have examined the role of vancomycin resistance on the outcome of enterococcal infections. Results have varied, with several demonstrating an association between vancomycin resistance and higher mortality. A 2003 meta-analysis of studies comparing outcomes in VRE and VSE bacteremia reported that, though most individual studies were insufficiently powered or adjusted to make this judgment, the available data suggested the VRE infections conferred higher mortality [7]. Few studies, however, have closely monitored patients' clinical courses throughout the hospital stay. We therefore investigated the systemic inflammatory response, clinical course and outcome of enterococcal BSI, stratifying by vancomycin susceptibility.

To characterize the severity of the patients' clinical condition and inflammatory response throughout the course of infection, we measured APACHE II scores and SIRS criteria from two days prior through 14 days after the onset of enterococcal BSI. Through univariate analysis, we found that patients' clinical state at onset of BSI influenced their mortality; patients with APACHE II>18 did significantly worse than those with APACHE $I I<18$, (figure 2). When stratifying for vancomycin-susceptibility, there was no difference in APACHE II scores on the day of infection or the two days preceding infection between the VRE and VSE groups. Despite the similar clinical state at onset of infection, the VRE group had a greater inflammatory response that peaked later in hospitalization than the VSE group. Maximal inflammatory response was seen on day 2 for the
VSE group and day 8 for the VRE group, (figure 3). Progression to septic shock was significantly associated with 7 -day and overall mortality. The greater inflammatory response of VRE species, however, did not translate into different outcomes as there was no statistical difference in incidence of organ failure, 7-day mortality or overall mortality. Our study showed non-significant trends toward increased mortality in the VRE group, and it is possible that with the increased power of a larger sample size, the greater inflammatory response would have correlated with increased mortality.

The enterococcal isolates in our study were predominantly E. faecalis and E. faecuim. The vancomycin resistance pattern followed speciation, with all of the E. faecalis isolates being vancomycin susceptible and all of the $E$. feacium isolates being vancomycin resistant. This is somewhat divergent from national surveys which report $60 \%$ vancomycin-resistance in E. faecium isolates. [1] Additionally, this raises the possibility that other factors innate to the particular species, besides antimicrobial resistance pattern, could influence the clinical course in our analysis. Earlier investigations have associated E. faecium with higher mortality than E. faecalis; either because it tends to colonize and infect sicker patients or because it is inherently more virulent. [15] In a study controlling for species by comparing VRE vs. VSE in E. faecium species alone, no increase in mortality was associated with vancomycin resistance [9].

Similar to other studies, our analysis demonstrated longer hospitalization was a risk factor for VRE infection, with mean length of stay of 30 days in the VRE group and 16 days in the VSE group. Age, sex, prior antibiotic use, mechanical ventilation, hemodialysis, stay in intensive care setting, central venous catheter use and neutropenia were not significant risk factors for vancomycin resistance in this study. 


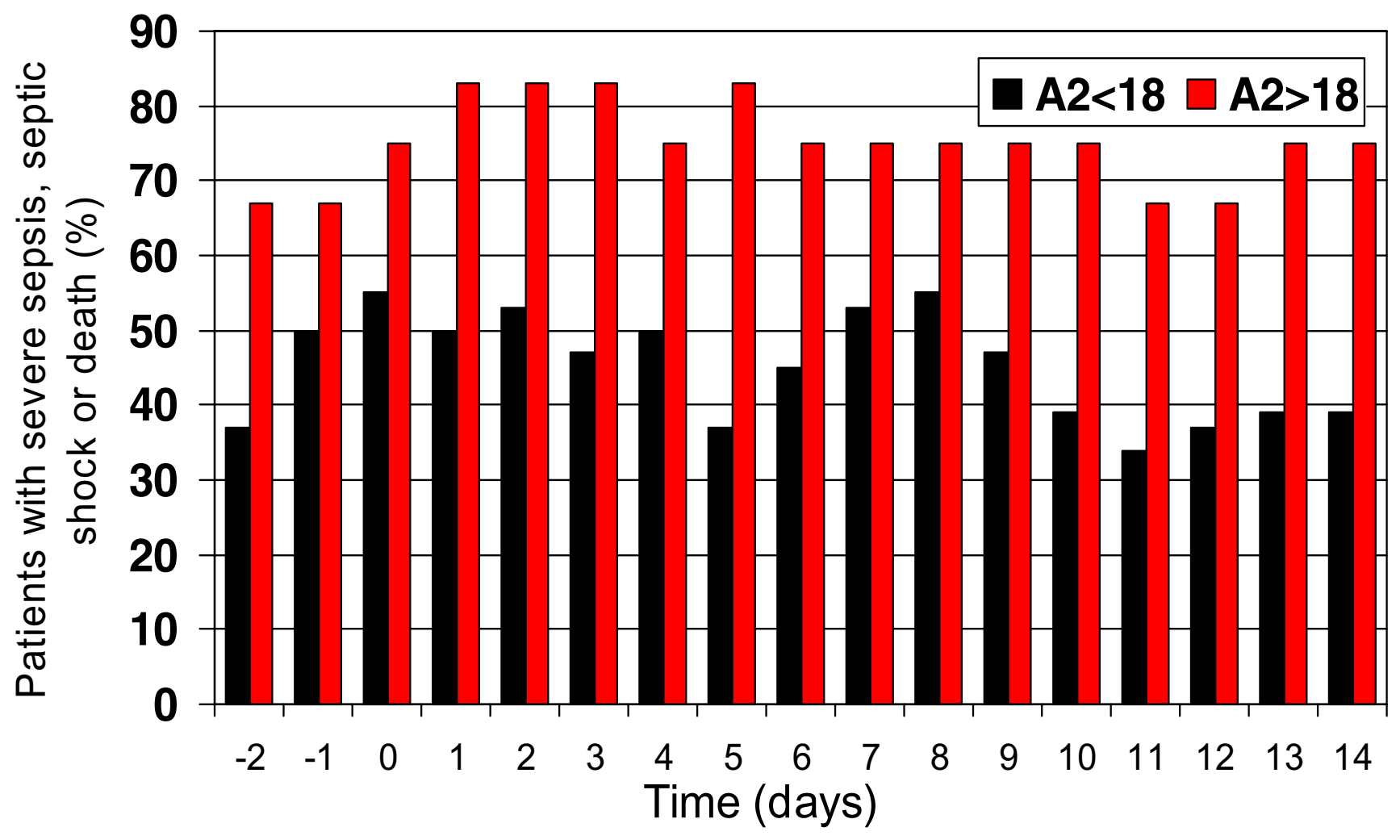

Figure 2

Severe sepsis, septic shock and death in patients with enterococcal BSI stratified by APACHE II score.

Appropriate antimicrobial therapy within 24 hours of infection was not a significant determinant of mortality. This differs from past studies, where appropriate antimicrobials were more rapidly used in VSE infections. This likely reflects the evolution of available antimicrobials with linezolid and quinupristin/dalfopristin being available during this study period, but not, or only on an investigational or compassionate-use-basis, in most earlier studies. These newer treatment options may change the mortality associated with VRE bacteremia. The more severely ill patients (those with APACHE II>18), did receive appropriate antimicrobial therapy significantly sooner ( 0.8 days versus 1.6 days), perhaps reflecting more aggressive clinical care or broader antibiotic coverage.

The presence of organ system failure was measured to further evaluate clinical course. Univariate analysis revealed that all measured forms of organ failure (respiratory, cardiovascular, renal, hematologic and liver) were associated with death. Multivariate analysis revealed that hematologic and cardiovascular failure independently predicted death. It is notable that hematologic failure, as defined by Fagon [13], includes the criterion of platelets $<100,000 /$ $\mu \mathrm{L}$. This may have been affected by use of linezolid, which is associated with thrombocytopenia and may confound this result [16].

This study failed to associate an increased inflammatory response in VRE BSI to significantly increased mortality. The underlying clinical state was significant in determining outcome, whereas the resistance pattern of the infecting organism and appropriate antimicrobial therapy were not. This supports other studies in which the enterococcal BSI were found to have insignificant impact on clinical outcome. These results have been attributed to the low virulence of enterococcal species. However, similar results were found in several recent studies with common methodology analyzing more virulent pathogens (Staphylococcus aureus, Candida spp and Pseudomonas aeruginosa). In these analyses, the underlying clinical condition (as measured by APACHE II score) was also significant, while the resistance pattern or appropriate antimicrobial therapy 


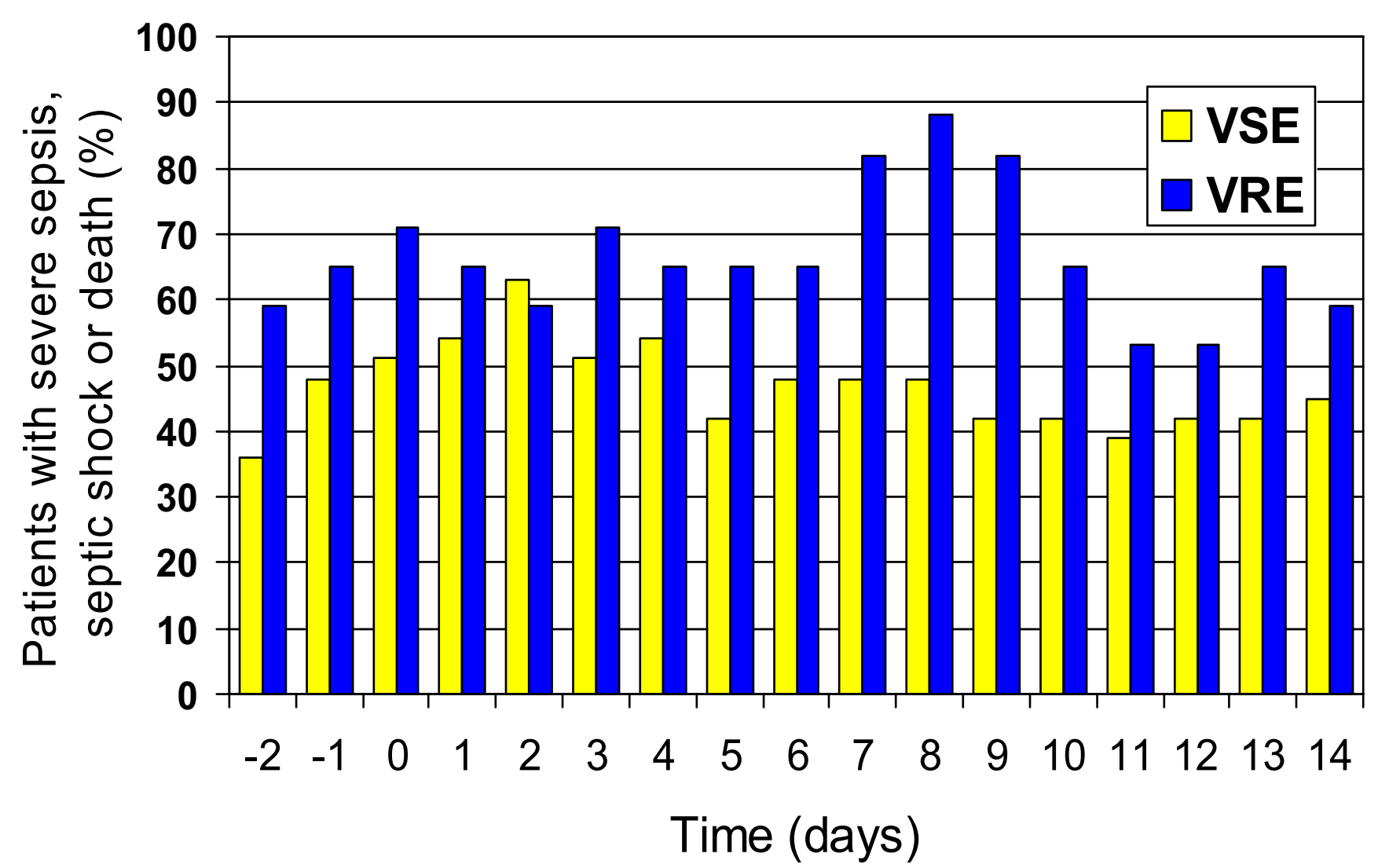

Figure 3

Severe sepsis, septic shock and death in patients with enterococcal BSI stratified by vancomycin resistance pattern.

were not. [17-19]. This furthers the concept that the host, rather than the pathogen, more greatly affects clinical outcome.

\section{Conclusion}

The overall morbidity and mortality of patients with enterococcal BSI is high. Morbidity correlates closely with the clinical condition of patient at the time of infection as measured by APACHE II score, regardless of vancomycin susceptibility pattern. Though showing a trend toward

Table 3: Risk factors for mortality in patients with enterococcal BSI

\begin{tabular}{lcccc}
\hline & \multicolumn{3}{c}{ Univariate analysis } & \multicolumn{2}{c}{ Multivariate analysis } \\
\hline & OR & $P$ & OR & $P$ \\
\hline Apache II score>18 & 4.6 & 0.03 & 3.2 & 0.28 \\
Respiratory failure & 8.0 & 0.005 & 3.6 & 0.15 \\
Cardiovascular failure & 9.7 & 0.002 & 7.5 & 0.03 \\
Renal failure & 3.9 & 0.049 & 0.8 & 0.88 \\
Hematologic failure & 6.4 & 0.01 & 8.4 & 0.02 \\
Liver failure & 5.9 & 0.015 & 3.3 & 0.26 \\
\hline
\end{tabular}

greater and later peaking inflammatory state, vancomycin resistance did not predict outcome. Timely treatment with appropriate antimicrobials also did not predict outcome. However, hematologic and cardiovascular failure predicted death.

\section{Competing interests}

The author(s) declare that they have no competing interests.

\section{Authors' contributions}

$\mathrm{KB}$ conducted the data collection, participated in the statistical analysis and drafted the manuscript. MB conceived of the study, participated in statistical analysis and edited the manuscript. HW, RW and GB contributed to study design.

\section{References}

I. Wisplinghoff H, Bischoff T, Tallant S, Seifert H, Wenzel RP, Edmond $M B$ : Nosocomial bloodstream infections in US hospitals: Analysis of 24,179 cases from a prospective nationwide surveillance study. Clinical Infect Dis 2004, 39:309-17.

2. Vergis EN, Hayden MK, Chow JW, Snydman DR, Zervos MD, Linden PK, Wegener MM, Schmitt B, Muder RR: Determinants of vanco- 
mycin resistance and mortality rates in enterococcal bacteremia. Ann Intern Med 200I, I35:484-492.

3. Bhavnani SM, Drake JA, Forrest A, Deinhart JA, Jones RN, Biedenbach $\mathrm{DJ}$, Ballow $\mathrm{CH}$ : A nationwide, multicenter, case-control study comparing risk factors, treatment, and outcome for vancomycin-resistant and -susceptible enterococcal bacteremia. Diagnostic Microbiology and Infectious Disease 2000, 36: I 45- I 48.

4. Edmond MB, Ober JF, Dawson JD, Weinbaum dl, Wenzel RP: Vancomycin-resistant enterococcal bacteremia: natural history and attributable mortality. Clin Infect Dis 1996, 23(6): I 234-9.

5. DiazGranados CA, Jernigan JA: Impact of Vancomycin Resistance on Mortality among Patients with Neutropenia and Enterococcal Bloodstream Infection. J Infect Dis 2005, 191:588-95.

6. Lodise TP, McKinnon PS, Tam VH, Rybak MJ: Clinical Outcomes for Patients with Bacteremia Caused by Vancomycin-Resistant Enterococcus in a Level I Trauma Center. Clin Infect Dis 2002, 34:922-9.

7. Salgado $C D$, Farr BM: Outcomes associated with vancomycinresistant enterococci: a meta-analysis. Infect Control Hosp Epidemiol 2003, 24(9):690-8.

8. Pelz RK, Lipsett PA, Swoboda SM, Diener-West M, Powe NR, Brower RG, Perl TM, Hammond JM, Hendrix CW: Vancomycin-sensitive and vancomycin-resistant enterococcal infections in the ICU: attributable costs and outcomes. Intensive Care Med 2002, 28:692-697.

9. Garbutt JM, Ventrapragada M, Littenberg B, Mundy LM: Association between resistance to vancomycin and death in cases of Enterococcus faecium bacteremia. Clin Infect Dis 2000, 30:466-72.

10. Shay DK, Maloney SA, Montecalvo M, Baneriee S, Wormser GP, Arduino MJ, Bland LA, Jarvis WR: Epidemiology and mortality risk of vancomycin-resistant enterococcal bloodstream infections. J Infect Dis 1995, I 72(4):993-1000.

II. Lautenbach E, Belker WB, Brennan PJ: Enterococcal bacteremia: risk factors for vancomycin resistance and predictors of mortality. Infect Control Hosp Epidemiol 1999, 20(5):3 I8-23.

12. American College of Chest Physicians/Society of Critical Care Medicine Consensus Conference: definitions for sepsis and organ failure and guidelines for the use of innovative therapies in sepsis. Crit Care Med 1992, 20:864-74.

13. Fagon JY, Chastre J, Novara A, Medioni P, Gibert C: Characterization of intensive care unit patients using a model based on the presence or absence of organ dysfunctions and/or infections: the OSIN mode. Intensive Care Med 1993, 19:137-44.

14. CDC guidelines for the prevention and control of nosocomial infections. Am J Infect Control I 983, I I (I):26-7.

15. Noskin GA, Peterson LR, Warren JR: Enterococcus faecium and Enterococcus faecalis bactermia: acquisition and outcome. Clin Infect Dis 1995, 20(2):296-30I.

16. Gerson SL, Kaplan SL, Bruss JB, Le V, Arellano FM, Hafkin B, Kuter $D$ J: Hematologic effects of linezolid: summary of clinical experience. Antimicrob Agents Chemother 2002, 46(8):2723-6.

17. Marra AR, Bar K, Bearman GML, Wenzel RP, Edmond MB: Systemic inflammatory response syndrome in adult patients with nosocomial bloodstream infection due to Pseudomonas aeruginosa. J Infect . 2006 Jan 26

18. Wisplinghoff $H$, Seifert $H$, Wenzel RP, Edmond MB: Inflammatory response and clinical course of adult patients with nosocomial bloodstream infections caused by Candida spp. Clinical Microbiology \& Infection 2006, I 2:170.

19. Wisplinghoff H, Seifert H, Coimbra M, Wenzel RP, Edmond MB: Systemic inflammatory response syndrome in adult patients with nosocomial bloodstream infection due to Staphylococcus aureus. Clin Infect Dis 200I, 33:733-6.

20. Lucas GM, Lechtzin N, Puryear DW, Yau LL, Flexner CW, Moore RD: Vancomycin-resistant and vancomycin-susceptible enterococcal bacteremia: comparison of clinical features and outcomes. Clin Infect Dis 1998, 26(5): I | 27-33.

21. Joels CS, Matthews BD, Sigmon LB, Hasan R, Lohr CE, Kercher KW, Norton J, Sing RF, Heniford BT: Clinical characteristics and outcomes of surgical patients with vancomycin-resistant enterococcal infections. Am Surg 2003, 69(6):5 I4-9.

22. Zaas AK, Song X, Tucker P, Perl TM: Risk factors for development of vancomycin-resistant enterococcal bloodstream infection in patients with cancer who are colonized with vancomycin-resistant enterococci. Clin Infect Dis 2002, 35: I I39-46. 23. Garner JS, Jarvis WR, Emori TG, JHoran TC, Hughes JM: CDC definitions for nosocomial infections. Am J Infect Control 1988, 16:128-40.

24. Murray B: Vancomycin-resistant enterococcal infections. New England Journal of Medicine . March 9, 2000

\section{Pre-publication history}

The pre-publication history for this paper can be accessed here:

http://www.biomedcentral.com/1471-2334/6/145/pre pub
Publish with Bio Med Central and every scientist can read your work free of charge

"BioMed Central will be the most significant development for disseminating the results of biomedical research in our lifetime. "

Sir Paul Nurse, Cancer Research UK

Your research papers will be:

- available free of charge to the entire biomedical community

- peer reviewed and published immediately upon acceptance

- cited in PubMed and archived on PubMed Central

- yours - you keep the copyright

Submit your manuscript here:

http://www.biomedcentral.com/info/publishing_adv.asp 